Wright State University

CORE Scholar

International Symposium on Aviation

International Symposium on Aviation

Psychology - 2009

Psychology

2009

\title{
Assisting Air Traffic Control in Planning and Monitoring Continuous Descent Approach Procedures
}

A. van der Eijk

M. Mulder

M.M. (René) van Paassen

A. C. in 't Veld

Follow this and additional works at: https://corescholar.libraries.wright.edu/isap_2009

Part of the Other Psychiatry and Psychology Commons

\section{Repository Citation}

van der Eijk, A., Mulder, M., van Paassen, M., \& in 't Veld, A. C. (2009). Assisting Air Traffic Control in Planning and Monitoring Continuous Descent Approach Procedures. 2009 International Symposium on Aviation Psychology, 184-189.

https://corescholar.libraries.wright.edu/isap_2009/84

This Article is brought to you for free and open access by the International Symposium on Aviation Psychology at CORE Scholar. It has been accepted for inclusion in International Symposium on Aviation Psychology - 2009 by an authorized administrator of CORE Scholar. For more information, please contact library-corescholar@wright.edu. 


\title{
ASSISTING AIR TRAFFIC CONTROL IN PLANNING AND MONITORING CONTINUOUS DESCENT APPROACH PROCEDURES
}

\author{
A. van der Eijk, M. Mulder, M. M. (René) van Paassen, A. C. in 't Veld \\ Delft University of Technology, Faculty of Aerospace Engineering \\ Kluijverweg 1, 2629HS Delft, The Netherlands
}

\begin{abstract}
In advanced noise abatement procedures, the approach of an aircraft is optimized to reduce the noise on the ground. A drawback of many noise abatement procedures is that air traffic controllers are forced to increase spacing, leading to a significant reduction of runway capacity. A display, named the Time-Space Diagram, has been developed to assist controllers in metering, sequencing and merging aircraft flying noise abatement procedures such as the Continuous Descent Approach. Although initial tests were promising, it was recommended that the information could be enhanced by supporting common controller spacing techniques. The improved display was tested in an experiment, in both low and high traffic rate scenarios. Results indicate that the use of the new display results in a significant reduction of controller workload. It also led to a reduction of the number of instructions to the pilots, suggesting a reduced workload on the flight deck as well.
\end{abstract}

Noise abatement procedures can significantly reduce the noise impact of aircraft during approach (Clarke, 2000; Erkelens, 2002). Unfortunately, the decrease in runway capacity that accompanies Continuous Descent Approach (CDA) procedures restricts the implementation of these noise abatement procedures (Erkelens, 2002). The main reason for this decrease in runway capacity is the inability of air traffic controllers to accurately predict separation between aircraft decelerating at different rates (Reynolds, Reynolds, \& Hansman, 2005). As a consequence, to counterbalance the differences in approach time, the separation distances between aircraft performing these procedures are increased substantially (Erkelens, 2002).

Previous research showed that predictions of 4-Dimensional Trajectories (4DTs), shown on an additional display, could support controllers in providing separation during CDA procedures (Tielrooij, In 't Veld, Mulder, \& Van Paassen, 2008). This additional display, the Time-Space Diagram (TSD), shows predictions of 4DTs in two dimensions: the horizontal axis indicates the aircraft Along Track Distance (ATD) to the runway, while the vertical axis depicts the corresponding Estimated Time of Arrival (ETA) at that distance. In-trail separation between aircraft is then represented by the horizontal distance between two predictions. The initial experimental validation of the TSD demonstrated its potential value in metering, sequencing and merging aircraft in CDAs (Tielrooij et al., 2008). Controllers reported, however, a lack of support for their common spacing strategies. In addition, the TSD would not present conflicts between aircraft on a fixed route and aircraft that were being vectored.

This paper describes the improvements made to the TSD, and the results of a controller-in-the-loop experiment that was conducted to quantify the effects of this display on operator performance, workload and the safety of operation.

\section{The Time-Space Diagram}

To make use of $4 \mathrm{D}$ trajectory predictions (either sent to the ground or computed on the ground), they have to be presented to the controller in a meaningful way. The Plan View Display (PVD) gives the current positions of aircraft but is not suited to display each aircraft's future trajectory, because on the plan view these would all overlap. An additional tool could provide the controller with a visual presentation of the future trajectories of aircraft from the current moment down to the runway. The Time-Space Diagram (TSD) provides this visual presentation, see Figure 1.

For each aircraft, the TSD shows the ATD to the runway on the horizontal axis and the ETA on the vertical axis. The connecting line represents a prediction for the future trajectory of that particular aircraft. Each point on the line corresponds to the ATD to the runway versus the predicted time of arrival, at that distance. The slope of the prediction line is an indication for the ground speed of an aircraft. Aircraft flying at a lower groundspeed will have a steeper prediction line than aircraft flying at a higher groundspeed. The space between two prediction lines shows the separation between the two aircraft. The horizontal dimension of this space represents the difference in ATD, the longitudinal separation if these two aircraft are on the same track to the runway (which may not be the case). The vertical dimension of separation space indicates the separation in time. 


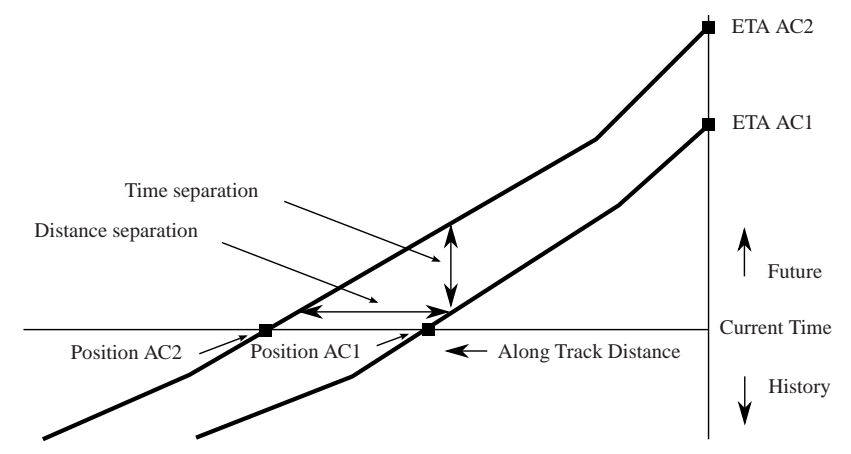

Figure 1: A schematic representation of the TSD (initial design)

Improving the TSD

One of the factors constraining the use of the initial TSD is the presentation of information(Tielrooij et al., 2008). The original and the improved versions are shown in Figure 2. The following changes were hypothesized to make the TSD more clear and self-explanatory.

\section{Layout of the TSD}

In the initial layout of the TSD the horizontal axis, representing the ATD, is located at the bottom of the screen, right above the routes, see Figure 2(a). According to the principle of pictorial realism, it is important that the diagram is analogous to the controller's mental model of the system and the physical system itself (Wickens, 1992). Placing the horizontal axis at the top of the screen (underneath the routes) and flipping the vertical axis results in a prediction line that suggests a descending aircraft. The slope of the prediction line is then compatible with the controller's mental model of an aircraft performing a CDA, even though the slope of the prediction line does not represent the vertical speed of the aircraft.

\section{Use of Transparency}

Subjects involved in the initial validation of the TSD reported that the representation of conflicts on the display was often considered problematic (Tielrooij et al., 2008). One of the main problems was overlap: when a conflict occurs, two separation areas overlap and on top of that a red conflict area will be drawn, making the individual predictions hard to distinguish. To solve this issue, transparency is introduced on the TSD. Separation areas and conflict areas are made transparent to a level that in case of a conflict, both the involved separation areas, the prediction line underneath and the conflict area on top, are visible.

\section{Use of Color}

On the initial TSD the use of color was limited: separation areas were colored light gray and conflict areas were colored red. All other objects were black and the background was gray. Color, however, could add another dimension to the TSD. In addition, colors could make the TSD more compatible with the PVD, thereby improving the mental model of the controller.

\section{Supporting Spacing Techniques on the TSD}

Part of this research was the analysis of the effects common spacing instructions have on the predictions shown on the TSD and how these common spacing strategies would solve a conflict (Tielrooij et al., 2008). The spacing strategies were divided into three categories based on their effects on the 4D trajectory: speed adjustment, changes to the planned route, and temporarily abandoning the planned route. 


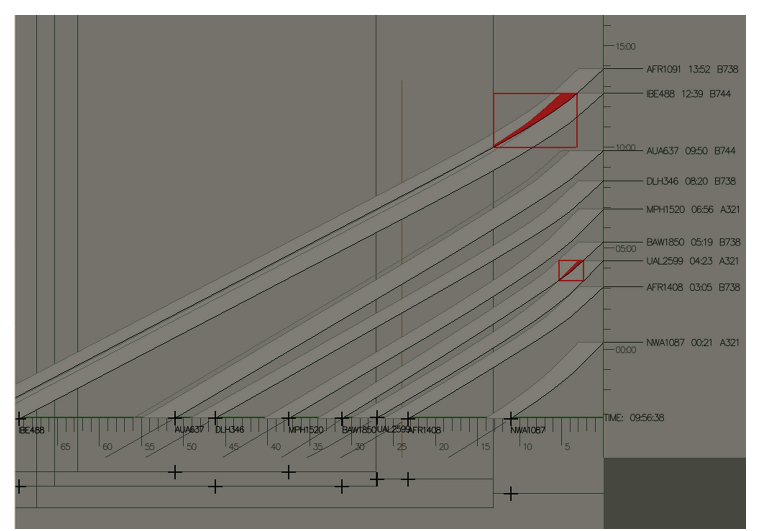

(a) original TSD

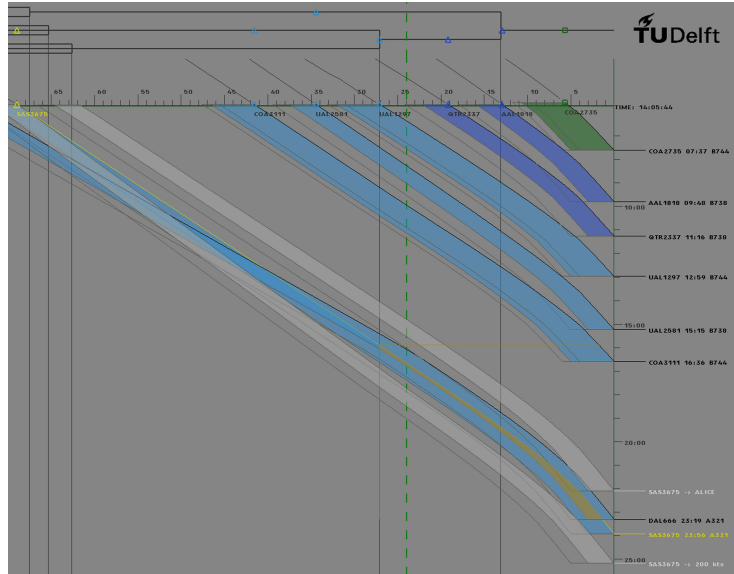

(b) improved TSD

Figure 2: Screenshots of the initial and the improved TSD.

\section{Speed Adjustment}

When two aircraft tend to become too close to each other, the controller can instruct the trailing aircraft to reduce its speed in order to increase separation. The rate at which spacing increases depends on the difference in speed between the leading and trailing aircraft. On the TSD, the slope of a prediction line indicates the speed of the aircraft, i.e., a speed reduction will lead to an increase in the slope. This results in an increase in ETA, while the ATD remains the same.

\section{Change to the Planned Route}

Another solution to increase separation between two aircraft is a change to the route of one of the aircraft. This can be done in two manners: 1) by giving the aircraft a direct instruction to a waypoint further down the route, or, 2) by giving the aircraft the instruction to enter a holding pattern. The waypoint instruction results in a decrease in the ATD, which means that the prediction will shift to the right on the TSD. The holding pattern instruction results in the addition of a specific amount of time to the ETA.

\section{Temporarily Abandoning the Planned Route}

Temporarily taking flights off the planned route is an often used strategy to increase separation. The pilot receives a heading instruction (vector) from the controller, which should be followed until enough separation is attained and the pilot is instructed to return to the planned route. However, when an aircraft deviates from its fixed lateral trajectory, the ATD is no longer defined. For the TSD, the ATD is then predicted at the present position and heading, assuming that the aircraft will return and continue the route at that point.

The advantage of this technique is that the controller will continuously receive an indication of the separation on the TSD. When a heading has been instructed by the controller, he/she can watch the TSD and instruct the aircraft to proceed with the approach when sufficient separation is predicted. The increase in ATD depends on two variables: moment of return and deviation angle. A large deviation angle increases the ATD amount of time. An early moment of return will lead to a small increase in ATD.

\section{Experiment}

To test the effects of the TSD an experiment has been performed in which controllers perform their task both with and without the additional display. The experiment further aimed at determining the effects of different scenarios on controller performance and workload. An important goal of the experiment was to receive feedback from controllers on the adapted display design. 
Subjects and Instructions Eight professional air traffic controllers participated in the experiment. The experience of the controllers ranged from 18 to 38 years. During an extensive briefing, subjects were instructed on the airspace, approach procedure, and the TSD. Subsequently, 90 minutes of training with different display configurations and traffic rates prepared the controllers for the actual experiment. The task of the subjects was to provide ATC services to approaching traffic while all aircraft performed a CDA procedure. To do this efficiently and safe, the subjects could use the following instructions: Speed reductions to a minimum of $180 \mathrm{kts}$ in steps of $10 \mathrm{kts}$, vectors, directs to a specified set of waypoints on the routes, and approach clearance.

Apparatus The apparatus is based on the air traffic simulation in(Tielrooij et al., 2008). For this project not only the TSD was further developed, the ATC simulator was improved as well.

Aircraft, Airspace and Atmosphere Point mass models of three different aircraft types were used: Airbus A321-100, Boeing B737-800 and B747-400, all at their maximum landing weights. To prevent the controllers from applying routine approach operations, learned through years of training and experience with a particular airspace, the airspace had to be unfamiliar. The airspace, airport and routes in the simulator were loosely based on the situation at Sydney's Kingsford Smith Airport, Australia. Approach routes were adapted to provide merging points and space for controller actions. The atmosphere was simulated as an International Standard Atmosphere (ISA) using the ISA relations up to $20,000 \mathrm{~m}$ and a logarithmic wind profile.

Independent Variables Two independent variables were defined: Display configuration (three levels: baseline PVD, PVD+TSD, and PVD+TSD ${ }^{+}$) and traffic rate (three levels: 15 aircraft per hour, 30 aircraft per hour, and 35 aircraft per hour).

Experiment Design and Procedure After the briefing and training, the measurement phase started. The measurement phase consisted of three blocks; one block for each display configuration. The blocks were randomized using a Latin square matrix to eliminate learning and boredom effects. To further cancel out these effects, the scenarios within a block were randomized as well and breaks were added in between. Before the start of each scenario, controllers could observe the traffic for a while to adjust to the scenario. After each scenario, subjects had to fill in a NASA Task Load indeX (TLX) (Hart \& Staveland, 1988) form. At the end of the entire experiment a questionnaire was completed by the subjects.

Dependent Measures The effects of the TSD were evaluated in terms of: 1) performance, measured by the difference between ETA and ATA and the number of instructions given by the controller to the blip driver, 2) safety, measured by the the number of conflicts (loss of separation), and 3) workload, measured using NASA TLX.

Results

\section{Performance}

Delay Figure 3 shows the results for the average delay per aircraft. ANOVA results for the complete experiment show that the delay per aircraft was not significantly affected by an increase in traffic rate, $F_{2,12}=2.240$, $p>0.05$. ANOVA results also show that no significant effect was found from the type of display on the delay per aircraft, $F_{2,14}=0.117, p>0.05$.

Instructions Figure 4 shows the results for the number of instructions per aircraft. ANOVA results show that with an increase in traffic rate, the number of instructions per aircraft did not significantly change, $F_{2,12}=1.250$, $p>0.05$. Mauchly's test indicated that the assumption of sphericity had been violated for the effects of display configuration, $\chi^{2}(2)=7.680, p<0.03$. Therefore degrees of freedom were corrected using Greenhouse-Geisser estimates of sphericity $(\epsilon=0.581)$. The results show that the number of instructions per aircraft were significantly affected by the display configuration, $F_{1.161,8.130}=5.271, p<0.05$. A post-hoc test revealed that this effect can be found between two groups, the cases with additional display (TSD and TSD ${ }^{+}$) and without additional display (PVD). 


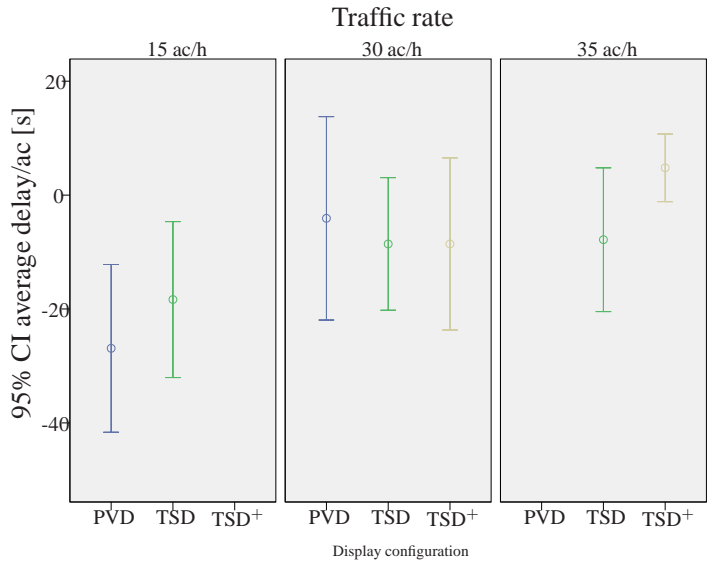

(a) Means and $95 \%$ confidence intervals

Figure 3: Results for the amount of delay

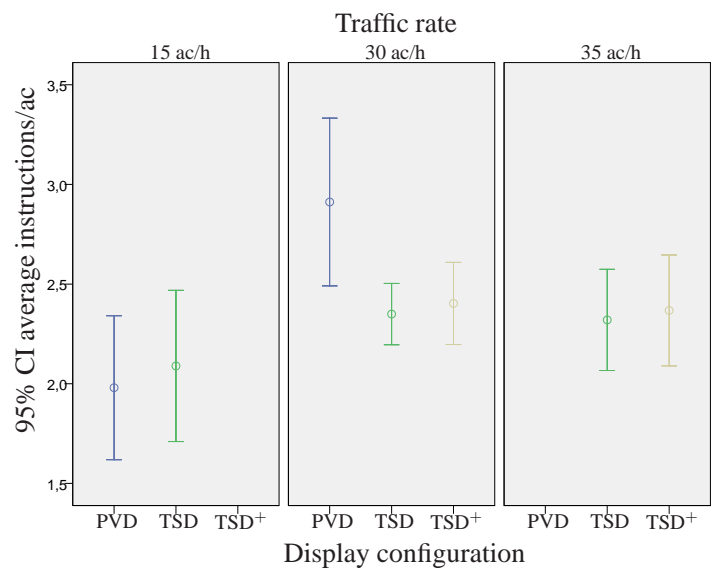

(a) Means and $95 \%$ confidence intervals

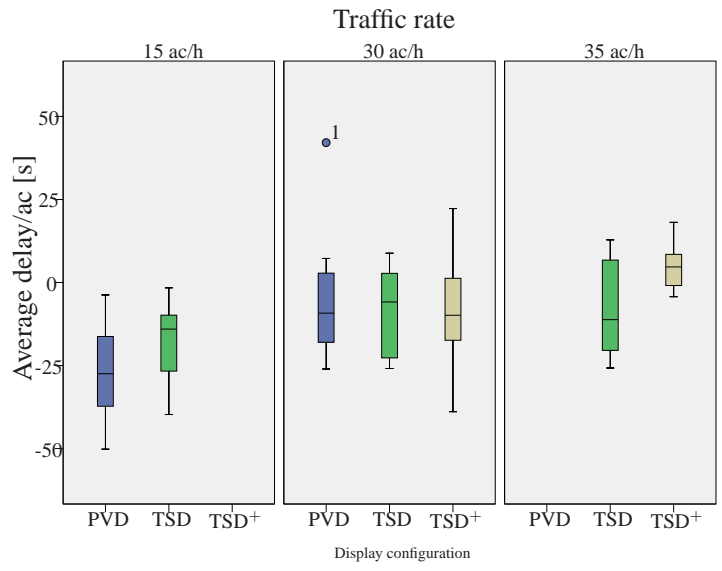

(b) Boxplots

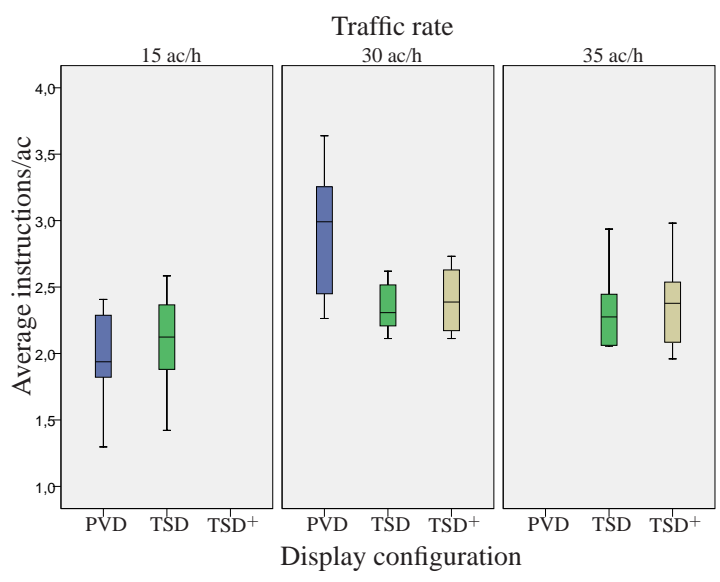

(b) Boxplots

Figure 4: Results for the number of instructions

Safety

During the experiment only three actual conflicts occurred. Two of these, however, were caused by bugs in the simulator software, related to unstable predictions. On the TSD, the aircraft seemed to be in conflict, while on the PVD there was sufficient separation. Therefore, these two conflicts could be neglected. Hence, the results on safety indicate that the display configuration will have no influence on the amount of conflicts.

\section{Workload}

Figure 5 shows the results for the z-score of the weighted NASA TLX subjective workload assessment. ANOVA results show that an increase in traffic rate had a highly significant effect on the experienced workload, $F_{2,12}$ $=25.090, p<0.01$. A post-hoc test indicated that this effect can be found between the low $(15 \mathrm{ac} / \mathrm{h})$ and high traffic rates (30 and $35 \mathrm{ac} / \mathrm{h})$. ANOVA results also show that with the change of display configuration the workload significantly decreased, $F_{2,14}=9.944, p=0.02$. A post-hoc test revealed that this effect can be found between two groups, the cases with additional display (TSD and $\mathrm{TSD}^{+}$) and without additional display (PVD). 


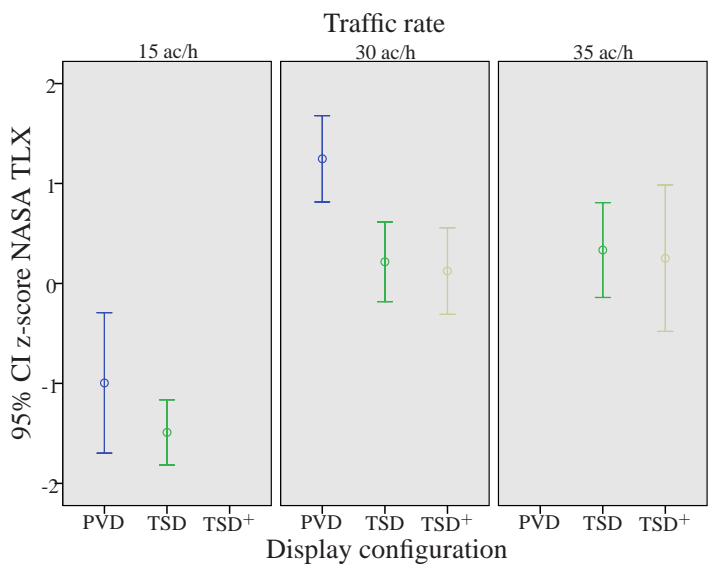

(a) Means and $95 \%$ confidence intervals

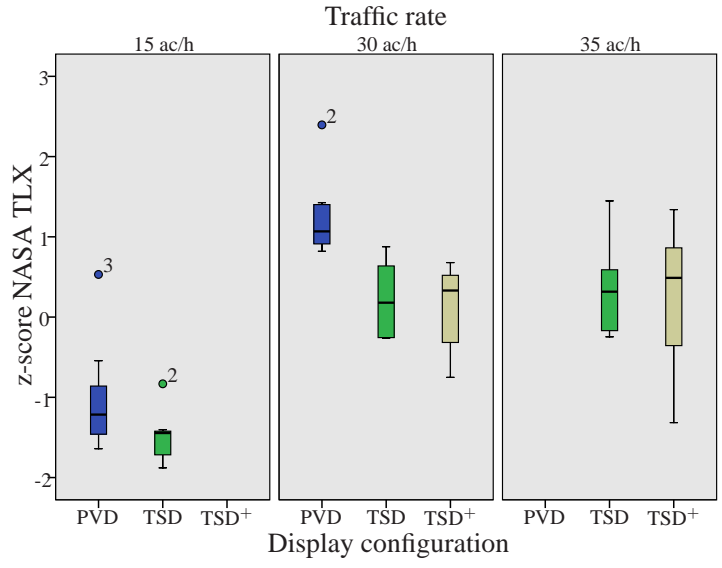

(b) Boxplots

Figure 5: Results of the normalized NASA TLX ratings

\section{Conclusions}

By depicting the along track distance versus the estimated time of arrival, the Time-Space Diagram uses 4dimensional trajectory predictions to present the in-trail separation between aircraft. An experiment was conducted to analyze the effects of the Time-Space Diagram on safety, controller performance and workload. Results show that the Time-Space Diagram significantly reduces controller workload and number of required instructions per aircraft for CDA procedures. No significant effects were found on delay and safety. The availability of additional predictions representing hypothetical instructions were found to have no significant results on the performance, safety and controller workload. Controllers who used these hypothetical predictions, however, stated that planning became easier.

\section{References}

Clarke, J.-P. B. (2000). Systems Analysis of Noise Abatement Procedures Enabled by Advanced Flight Guidance Technology. Journal of Aircraft, 37(2).

Erkelens, L. J. J. (2002). Advanced Noise Abatement Procedures for Approach and Departure. In AIAA Guidance, Navigation, and Control Conference and Exhibit. Monterey, CA, USA.

Hart, S. G., \& Staveland, L. E. (1988). Development of NASA-TLX (Task Load Index): Results of Empirical and Theoretical Research. In Human Mental Workload (pp. 139-183). Amsterdam, The Netherlands: Elsevier Science Publishers (North Holland).

Reynolds, H. J. D., Reynolds, T. G., \& Hansman, R. J. (2005). Human Factors Implications of Continuous Descent Approach Procedures for Noise Abatement in Air Traffic Control. In 6th USA/Europe Air Traffic Management Research and Development Seminar. Baltimore, WA, USA.

Tielrooij, M., In ‘t Veld, A. C., Mulder, M., \& Van Paassen, M. M. (2008). Assisting ATC in Metering, Sequencing and Merging Arrival Streams in Three-Degree Decelerating Approaches. Proceedings of the 27th European Annual Conference on Human Decision Making and Manual Control, Delft, The Netherlands, June 12-13.

Wickens, C. D. (1992). Engineering Psychology and Human Performance. New York, NY, USA: Harper Collins Publishers Inc. (Second edition) 